\section{SANDIA REPORT}

SAND96-2268 - UC-910

Unlimited Release

Printed September 1996
RECEIVED

SEP 30 1996

OSTI

\title{
Analysis of Waveguiding Properties of VCSEL Structures
}

Ireena A. Erteza

\author{
Prepared by \\ Sandia National Laboratories \\ Albuquerque, New Mexico 87185 and Livermore, California 94550 \\ for the United States Department of Energy \\ under Contract DE-AC04-94AL85000
}

Approved for public release; distribution is unlimited 


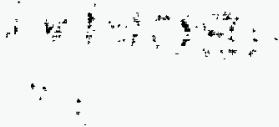

Issued by Sandia National Laboratories, operated for the United States Department of Energy by Sandia Corporation.

NOTICE: This report was prepared as an account of work sponsored by an agency of the United States Government. Neither the United States Government nor any agency thereof, nor any of their employees, nor any of their contractors, subcontractors, or their employees makes any warranty, express or implied, or assumes any legal liability or responsibility for the accuracy, completeness, or usefulness of any information, apparatus, product, or process disclosed, or represents that its use would not infringe privately owned rights. Reference herein to any specific commercial product, process, or service by trade name, trademark, manufacturer, or otherwise, does not necessarily constitute or imply its endorsement, recommendation or favoring by the United States Government, any agency thereof or any of their contractors or subcontractors. The views and opinions expressed herein do not necessarily state or reflect those of the United States Government, any agency thereof or any of their contractors.

Printed in the United States of America. This report has been reproduced directly from the best available copy.

Available to DOE and DOE contractors from

Office of Scientific and Technical Information

PO Box 62

Oak Ridge, TN 37831

Prices available from (615)576-8401, FTS 626-8401

Available to the public from

National Technical Information Service

US Department of Commerce

5285 Port Royal Rd

Springfield, VA 22161

NTIS price codes

Printed copy: A03

Microfiche copy: A01 


\section{DISCLAMMER}

Portions of this document may be illegible in electronic image products. Images are produced from the best available original document. 
SAND96-2268

Unlimited Release

Distribution

Printed September 1996

Category UC-910

\title{
Analysis of Waveguiding Properties of VCSEL Structures
}

\author{
Ireena A. Erteza \\ Exploratory Systems Development Center \\ Sandia National Laboratories \\ Albuquerque, NM 87185-0843
}

\begin{abstract}
In this paper, we explore the feasibility of using the distributed Bragg reflector, grown on the substrate for a VCSEL (Vertical Cavity Surface Emitting Laser), to provide waveguiding within the substrate. This waveguiding could serve as an interconnection among VCSELs in an array.

Before determining the feasibility of waveguide interconnected VCSELs, two analysis methods are presented and evaluated for their applicability to this problem. The implementations in Mathematica of both these methods are included.

Results of the analysis show that waveguiding in VCSEL structures is feasible. Some of the many possible uses of waveguide interconnected VCSELs are also briefly discussed. The tools and analysis presented in this report can be used to evaluate such system concepts and to do detailed design calculations.
\end{abstract}




\section{Acknowledgements}

This work was supported by the United States Department of Energy under Contract DE-AC04-94AL85000. 


\section{Contents}

1 Introduction 1

2 Analysis Methods 1

2.1 Electromagnetic Analysis of Propagation in a Periodic Media- the Bloch Wave Approach . . . . . . . . . . . . . . 2

2.1 .1 TM modes . . . . . . . . . . . . . 6

2.2 Field Transfer Matrix Approach for Planar Multilayer Waveguides . 6

2.2.1 Bound Modes . . . . . . . . . . . . . . 8

2.2.2 Radiation Modes ............... 8

2.2.3 Leaky Modes ................. 8

3 Comparison and Appropriateness of the Analysis Methods 9

4 Bloch Wave Implementation $\quad 10$

5 Field Transfer Matrix Approach $\quad 14$

6 Examples of Confined Lossless Modes in a VCSEL Structures 15

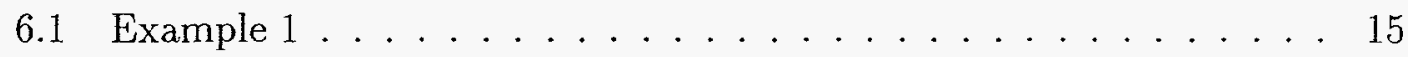

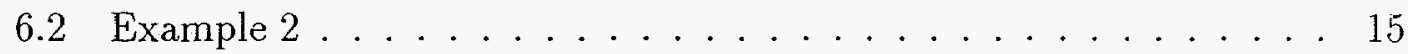

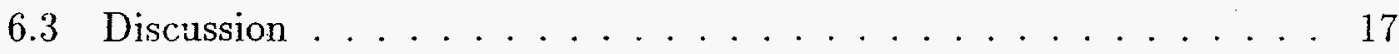

7 Waveguide Interconnected VCSELs $\quad 17$

8 Conclusion $\quad 20$

$\begin{array}{lr}\text { A Bloch Wave Method } & 20\end{array}$

B Field Transfer Matrix Approach $\quad 22$

B.1 Explicit Layer Definition . . . . . . . . . . . . . . . 22

B.2 Periodic Layer Definition . . . . . . . . . . . . 26 


\section{List of Figures}

1 Simplified VCSEL Structure . . . . . . . . . . . 2

2 Periodic Medium . . . . . . . . . . . . . . . . 3

3 Bragg Reflection Waveguide . . . . . . . . . . . . 4

4 Thin Film Waveguide . . . . . . . . . . . . . . 7

$5 \quad$ Plot of $\left|\frac{A+D}{2}\right|-1$ vs. $k \beta \ldots \ldots \ldots \ldots \ldots \ldots$

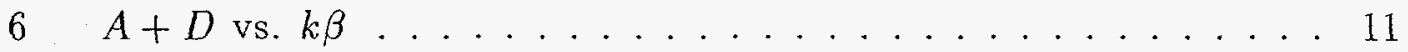

$7 \quad$ Plot of the right hand side of the dispersion equation vs. $k \beta \ldots \ldots 12$

8 Combined plot of the RHS and LHS of the dispersion equation as a function of guide thickness for $k \beta=3.44 \ldots \ldots \ldots 13$

9 Combined plot of the RHS and LHS of the dispersion equation as a function of guide thickness for $k \beta=3.2 \ldots \ldots \ldots 14$

10 Solutions to the dispersion equation for the structure described in Example 1 and a guide thickness of $100 \mu \mathrm{m} . \ldots \ldots \ldots$

11 Solutions to the dispersion equation for a) $\lambda=.98 \times 10^{-6}$; b) $\lambda=$ $.99 \times 10^{-6}$; and c) $\lambda=1.3 \times 10^{-6}$ for guide thickness of $100 \mu \mathrm{m} . \quad .19$ 


\section{Introduction}

Recent progress in molecular beam epitaxy (MBE) has resulted in the ability to grow a variety of novel devices. One such device that is receiving much attention is the Vertical Cavity Surface Emitting Laser (VCSEL). Because of the small cavity size, the gain length in a VCSEL is short. This necessitates a mirror with high reflectivity in order for the VCSEL to reach threshhold. For ease in processing, the high reflectance mirrors in VCSELs are typically semiconductor distributed Bragg reflector (DBR) mirrors. While such mirrors are critical to the functioning of the VCSEL device, they might also play another role in an interconnected system of VCSELs.

In this paper, we explore the feasibility of using the DBR grown on the substrate for a VCSEL to provide waveguiding within the substrate. This waveguiding could serve as an interconnection among VCSELs in an array.

Before this system concept can be analyzed, the waveguiding properties of a DBR grown on a substrate must be studied. There are two approaches which can be taken for this analysis. One is a standard thin film analysis of planar multilayer waveguides. The other is an analysis employing an understanding of wave propagation in periodic media. As the DBR is made up of approximately 25 quarter wave pairs, it can be considered a periodic medium. Since both these approaches appear at first look to be applicable, we will present both of the approaches and explain their appropriateness to the problem of interest.

With the analysis methods explained, we will then present some results from the analysis. These results will be discussed, and some general ideas of how waveguiding in VCSEL structures can be used in systems will be presented.

The analysis methods and tools presented and developed here can be used to make design calculations for any system concept using waveguiding in VCSEL structures. Issues such as epitaxial tolerances, distances and materials can also be addressed. This will be the subject of a subsequent report.

\section{Analysis Methods}

To determine which analysis methods are appropriate to find the waveguiding properties of VCSEL structures, we must first get a clear understanding of what we want to analyze.

Consider a typical VCSEL structure. A simplified schematic is shown in Figure 1. We are interested in perhaps guiding the emitted laser light within the substrate by coupling it into an appropriate mode via a diffractive optical element (DOE) on the substrate/air interface.

For our purposes, there are several things required from an analysis of the air- 


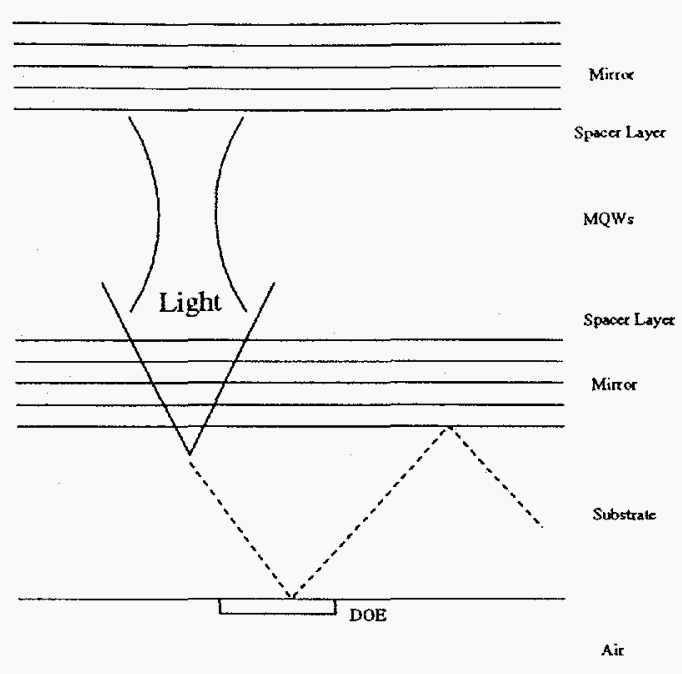

Figure 1: Simplified VCSEL Structure

substrate-DBR structure. First, it must be determined whether or not a mode can be guided in the substrate. Then, the propagation constant of the guided modes (if any) need to be found. Also, the loss associated with any propagating modes must be calculated. Based on these results, the feasibility of interconnecting VCSELs using these waveguides can be assessed.

There are two analysis approaches for this type of structure: (1) the electromagnetic analysis of propagation in a periodic media [YY84, Yeh88]; and (2) the field transfer matrix approach for planar multilayer waveguides [BW75, CH84]. In the following sections, these two methods will be described. The application and appropriateness of each method to the problem of interest will also be discussed.

\subsection{Electromagnetic Analysis of Propagation in a Peri- odic Media- the Bloch Wave Approach}

This method follows the standard modal analysis of planar waveguides [Mar91], with the modification of the field form in the periodic cover layer to accomodate Bloch waves. [YYH77]

To review, the Bloch wave function is the form a propagating wave in a periodic medium takes, according to the Floquet theorem [Blo28]. A Bloch wave propagating in the $z$ direction (in a medium periodic in $x$ ) is given by

$$
E(x, z)=E_{K}(x) e^{i K x} e^{i \beta z}
$$

where $K$ is a constant, called the Bloch wave number, and $E_{K}(x)$ is periodic, with the same period as the periodic media. The periodic medium is shown in Figure 2. Each repeating period is referred to as a unit cell.

The equations for a Bloch wave are derived using the matrix method and translation operator applied to a periodic medium. Details can be found in Refer- 


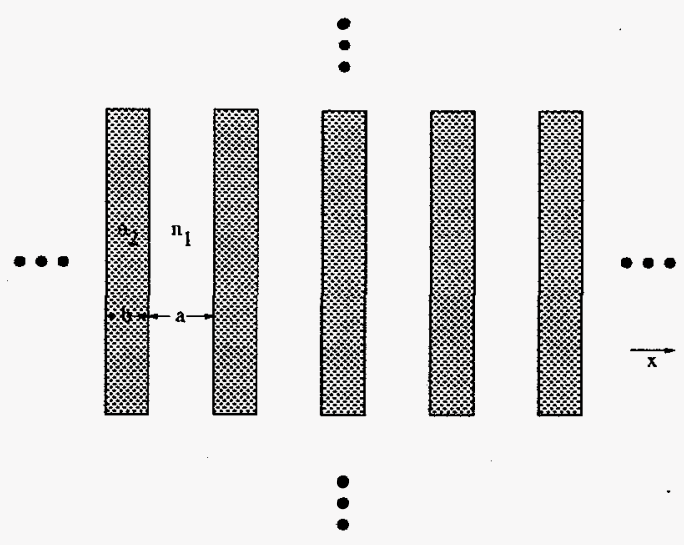

Figure 2: Periodic Medium

ence [YYH77].

The following equations are used in describing a TE Bloch wave.

$$
\begin{gathered}
A=e^{-i k_{1 x} a}\left[\cos \left(k_{2 x} b\right)-\frac{i}{2}\left(\frac{k_{2 x}}{k_{1 x}}+\frac{k_{1 x}}{k_{2 x}}\right) \sin \left(k_{2 x} b\right)\right] \\
B=e^{i k_{1 x} a}\left[-\frac{i}{2}\left(\frac{k_{2 x}}{k_{1 x}}-\frac{k_{1 x}}{k_{2 x}}\right) \sin \left(k_{2 x} b\right)\right] \\
C=B^{*}=e^{-i k_{1 x} a}\left[\frac{i}{2}\left(\frac{k_{2 x}}{k_{1 x}}-\frac{k_{1 x}}{k_{2 x}}\right) \sin \left(k_{2 x} b\right)\right] \\
D=A^{*}=e^{i k_{1 x} a}\left[\cos \left(k_{2 x} b\right)+\frac{i}{2}\left(\frac{k_{2 x}}{k_{1 x}}+\frac{k_{1 x}}{k_{2 x}}\right) \sin \left(k_{2 x} b\right)\right] \\
e^{-i K \Lambda}=\frac{1}{2}(A+D) \pm \sqrt{\left[\frac{(A+D)}{2}\right]^{2}-1} \\
k_{1 x}=\sqrt{\left(\frac{w n_{1}}{c}\right)^{2}-\beta^{2}} \\
k_{2 x}=\sqrt{\left(\frac{w n_{2}}{c}\right)^{2}-\beta^{2}} \\
a_{0}=B \\
b_{0}=e^{-i K \Lambda}-A
\end{gathered}
$$

The Bloch wave in the layer with index $n_{1}$ in the $n^{\text {th }}$ unit cell of the periodic medium is

$$
E(x)=E_{K}(x) e^{i K x}=\left[\left(a_{0} e^{i k_{1 x}(x-n \Lambda)}+b_{0} e^{-i k_{1 x}(x-n \Lambda)}\right) e^{-i K(x-n \Lambda)}\right] e^{i K x}
$$

and the Bloch wave number can be written explicitly as

$$
K=\frac{1}{\Lambda} \arccos \left(\frac{A+D}{2}\right)
$$


$a_{0}$ and $b_{0}$ refer to the magnitude of an incident and reflected wave on layer $n_{1}$ in the first unit cell.

There are several points to note about Bloch waves. First, the waveform consists of a periodic function multiplied by $e^{i K x}$. Regions where

$$
\left|\frac{A+D}{2}\right|<1
$$

correspond to real $K$, and hence propagating Bloch waves.

Where $\left|\frac{A+D}{2}\right|>1, K$ is complex with a value of $K=\frac{m \pi}{\Lambda}+i K_{i} . K_{i}$ is the imaginary part of $K$, and $m$ is an integer. In these regions, the Bloch wave is evanescent. These are considered the forbidden bands of the periodic medium.

For a waveguide, as in Figure 3, with a low, constant index material as one cladding

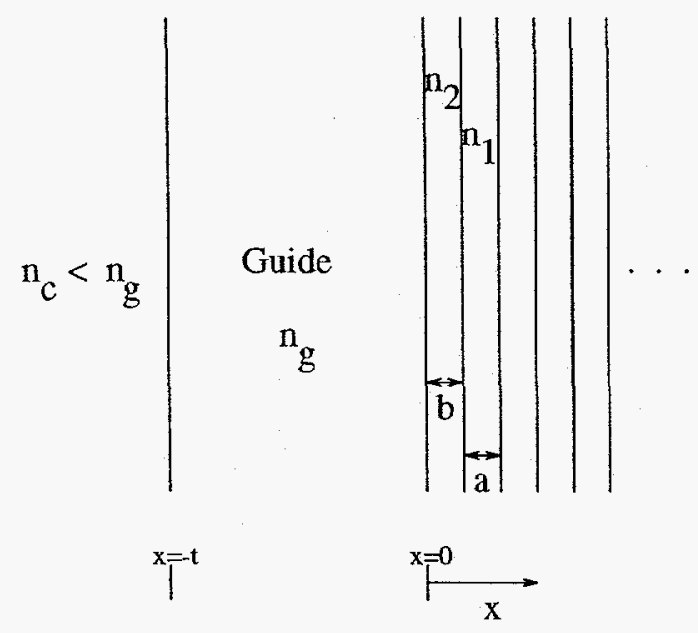

Figure 3: Bragg Reflection Waveguide

and a periodic index medium as the other cladding, the assumed solution for the electric field of the TE mode is:

$$
E(x)= \begin{cases}e^{q_{a}(x+t)}, & x<-t \\ c_{1} \cos \left(k_{g}(x+t)\right)+c_{2} \sin \left(k_{g}(x+t)\right), & -t \leq x<0 \\ E_{K}(x) e^{i K x}, & 0 \leq x\end{cases}
$$

where

$$
q_{a}=\sqrt{\beta^{2}-\left(\frac{\omega n_{a}}{c}\right)^{2}}
$$

and

$$
k_{g}=\sqrt{\left(\frac{\omega n_{g}}{c}\right)^{2}-\beta^{2}}
$$

Continuity of the field and its derivative on the two interfaces, $x=0$ and $x=-t$ must be satisfied. This requires the following dispersion relation to be satisfied for 
TE modes.

$$
k_{g}\left(\frac{q_{a} \cos k_{g} t-k_{g} \sin k_{g} t}{q_{a} \sin k_{g} t+k_{g} \cos k_{g} t}\right)=-i k_{1 x} \frac{e^{-i K \Lambda}-A-B}{e^{-i K \Lambda}-A+B}
$$

For confined propagation in the guide, $\beta, q_{a}$ and $k_{g}$ are real, and the left-hand side of the dispersion equation is real. In general, the right-hand side of the dispersion equation is complex. It is only real when the Bloch wave is evanescent, or equivalently when the Bloch propagation constant is in a forbidden band. This propagation is lossless, since $\beta$ is real.

This type of confined and lossless mode propagation can also be explained in a more intuitive manner [YYH77]. In order for guiding, the mode must experience complete reflection at both interfaces. The reflection at the guide-periodic medium interfaces requires that the mode correspond to a forbidden gap in the periodic medium. In order to ensure that the mode propagates, the round trip phase delay in the guiding region must be a multiple of $2 \pi$.

In practice, the periodic medium is finite in extent, hence there will not be $100 \%$ reflectance at the guide-periodic medium boundary, resulting in loss. The effective attenuation can be estimated from the following rationale. [Yeh88]

The reflectivity, $R$, of a finite Bragg reflector with $N$ unit cells can be written as

$$
R=\frac{|C|^{2}}{|C|^{2}+\left(\frac{\sinh K_{i} \Lambda}{\sinh N K_{i} \Lambda}\right)^{2}}
$$

Let $\theta$ be the angle of incidence of the guided ray in the guide layer. With each round trip, the ray travels a distance of $2 t \tan \theta$ along the direction of propagation. Therefore, the number of roundtrips a ray makes while traveling a distance $L$ along the waveguide is

$$
\mathcal{N}=\frac{L}{2 t \tan \theta}
$$

The attenuation with each round trip is $R$, therefore the attenuation with $\mathcal{N}$ round trips is $R^{\mathcal{N}}$. Using the standard form for an attenuation constant, $\alpha$

$$
e^{-\alpha L}=R^{\mathcal{N}}
$$

then

$$
\alpha=-\frac{\ln R}{2 t \tan \theta}=\frac{-k_{g}}{2 \beta t} \ln R
$$

If $t$ is given in units of meters, then the attenuation in $\frac{\mathrm{dB}}{\mathrm{cm}}$ is given by

$$
\frac{2(4.34) \alpha}{100}
$$




\subsubsection{TM modes}

The equations for TM modes are the same as the TE mode equations, except for A, B, C, D and the dispersion equation. The appropriate equations are as follows.

$$
\begin{gathered}
A=e^{-i k_{1 x} a}\left[\cos \left(k_{2 x} b\right)-\frac{i}{2}\left(\frac{n_{2}^{2} k_{1 x}}{n_{1}^{2} k_{2 x}}+\frac{n_{1}^{2} k_{2 x}}{n_{2}^{2} k_{1 x}}\right) \sin \left(k_{2 x} b\right)\right] \\
B=e^{i k_{1 x} a}\left[-\frac{i}{2}\left(\frac{n_{2}^{2} k_{1 x}}{n_{1}^{2} k_{2 x}}-\frac{n_{1}^{2} k_{2 x}}{n_{2}^{2} k_{1 x}}\right) \sin \left(k_{2 x} b\right)\right] \\
C=B^{*} \\
D=A^{*} \\
k_{g}\left(\frac{n_{2}}{n_{g}}\right)^{2}\left(\frac{q_{a} \cos k_{g} t-k_{g} \sin k_{g} t}{q_{a} \sin k_{g} t+k_{g} \cos k_{g} t}\right)=-i k_{1 x} \frac{e^{-i K \Lambda}-A-B}{e^{-i K \Lambda}-A+B}
\end{gathered}
$$

\subsection{Field Transfer Matrix Approach for Planar Multi- layer Waveguides}

The second method proposed to analyze the waveguiding situation in Figure 1 is based on having a unimodular field matrix, $M_{j}$, to relate the field amplitudes $U_{j}, V_{j}$ at a distance $x_{j}$ to the corresponding amplitudes at another point, $x_{j-1}$. [CH84]

$$
\left[\begin{array}{l}
U_{j-1} \\
V_{j-1}
\end{array}\right]=M_{j}\left[\begin{array}{l}
U_{j} \\
V_{j}
\end{array}\right]
$$

The total electric or magnetic field waves are sums of positive and negative-going waves, and have the form

$$
\exp [i k( \pm \alpha x+\beta y)]
$$

where

$$
\begin{gathered}
\alpha=n \cos \theta=\sqrt{n^{2}-\beta^{2}} \\
\beta=n \sin \theta \\
k=\frac{2 \pi}{\lambda} \\
z 0=\sqrt{\frac{\mu}{\epsilon}}
\end{gathered}
$$

The geometry is illustrated in Figure 4.

For the TE case, with propagation in the $y$ direction, $U$ refers to $E_{z}$, and $V$ refers to $-H_{y}$. In this case, the unimodular transfer matrix is

$$
M_{j}=\left[\begin{array}{cc}
\cos \phi_{j} & -\frac{i}{\gamma_{j}} \sin \phi_{j} \\
-i \gamma_{j} \sin \phi_{j} & \cos \phi_{j}
\end{array}\right]
$$


where

$$
\begin{gathered}
\gamma_{j(T E)}=\frac{\alpha}{z_{o}} \\
\phi_{j}=k \alpha_{j}\left(x_{j}-x_{j-1}\right)=\text { phase thickness of the layer }
\end{gathered}
$$

For completeness, in the TM case (propagation in the $y$ direction) $U$ refers to $H_{z}$, and $V$ refers to $E_{y}$. The unimodular transfer matrix is the same as in Equation 34, with.

$$
\gamma_{j(T M)}=\frac{\alpha z_{o}}{n^{2}}
$$

For a stack of $J$ films, the field transfer matrices multiply,

$$
M=\prod_{j=1}^{J} M_{j}
$$

For a waveguide composed of a stack of thin films, as in Figure 4, the fields in the cover can be related to those in the substrate by

$$
\left[\begin{array}{l}
U_{C} \\
V_{C}
\end{array}\right]=M\left[\begin{array}{l}
U_{S} \\
V_{S}
\end{array}\right]
$$

where $M$ is the transfer matrix for the stack of films.

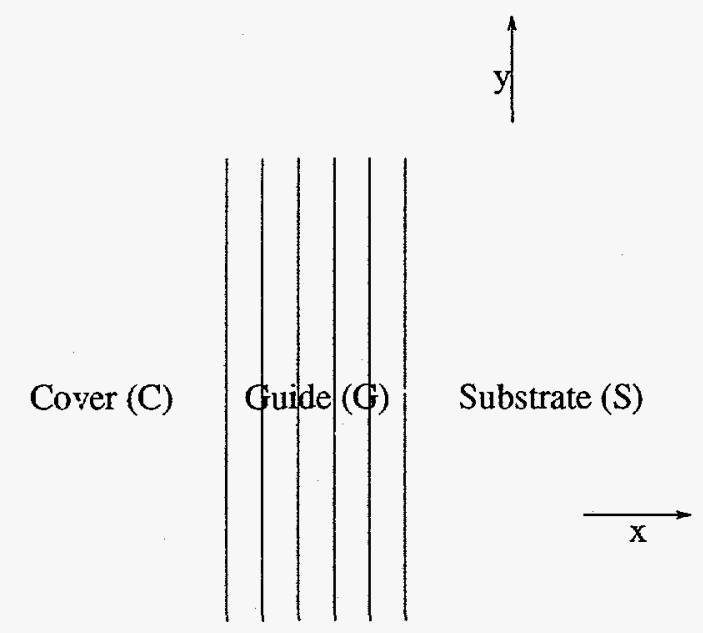

Figure 4: Thin Film Waveguide

Solutions to this situation fall into three categories:

- Bound Modes

- Radiaton Modes

- Leaky Modes 


\subsubsection{Bound Modes}

For bound modes, the fields in the cover and substrate must decay exponentially from the stack (i.e. the radiation condition must be satisfied). Consequently, the waves in the cover are negative-going waves, and those in the substrate are positive-going. Equation 39 becomes

$$
\left[\begin{array}{c}
-1 \\
\gamma_{C}
\end{array}\right] U_{C}=M\left[\begin{array}{c}
1 \\
\gamma_{S}
\end{array}\right] U_{S}
$$

Equivalently, the condition for a bound mode is

$$
\chi(\beta)=\gamma_{C} m_{11}+\gamma_{C} \gamma_{S} m_{12}+m_{21}+\gamma_{S} m_{22}=0
$$

Only a set of discrete roots (i.e. discrete $\beta$ values) satisfy the modal condition.

According to the propagation directions and conventions assumed earlier, Equation 41 requires $\alpha_{S}$ and $\alpha_{C}$ to have positive imaginary parts.

For a lossless dielectric waveguide, $\beta$ is real, and $\beta>n_{c}, n_{s}$. As a result, $\chi(\beta)$ is imaginary.

\subsubsection{Radiation Modes}

For radiation modes, the field in the cover and substrate is a standing wave. Technically, the mode could decay exponentially on one interface and be a standing wave on the other. For a full radiation mode (standing wave on both claddings), $\beta<n_{C}, n_{S}$. Equation 39 still is valid, but the modal condition, Equation 41 , is not satisfied. There is a continuum of $\beta$ values for radiation modes.

\subsubsection{Leaky Modes}

Leaky modes are extensions of the bound modes that occur below the cutoff of the standard bound mode. For leaky modes, the modal condition, Equation 41, is satisfied, but $\beta$ takes on complex values. Complex $\beta$ values for leaky modes are discrete.

The derivation for $\chi(\beta)$ in Equation 41 relied on the choice of positive-going or negative-going waves in the bounding media to satisfy the radiation condition. When $\beta$ is complex, it is only the inward-traveling (i.e. going toward the stack) wave that can satisfy the radiation condition. Therefore, for leaky modes, the assumptions for the waves in the cover and substrate are opposite the assumptions made for guided modes. Consequently, in order to use Equation $41, \alpha_{S}$ and $\alpha_{C}$ must have negative imaginary parts. Because the real and imaginary parts for any $\alpha$ in a lossless medium are of opposite sign, the previous condition is equivalent to requiring the real parts of $\alpha_{S}$ and $\alpha_{C}$ to be positive. 


\section{Comparison and Appropriateness of the Anal- ysis Methods}

The two methods described above are very similar in their approach of assuming general forms of the solutions for the fields, and then choosing appropriate constants to ensure that Maxwell's equations and boundary conditions are satisfied. The key difference, however, is in the assumed wave forms.

For the case shown in Figure 3, the assumed wave form in the periodic media for the first method is given by

$$
E(x, z)=E_{K}(x) e^{i K x}=\left[\left(a_{0} e^{i k_{1 x}(x-n \Lambda)}+b_{0} e^{-i k_{1 x}(x-n \Lambda)}\right) e^{-i K(x-n \Lambda)}\right] e^{i K x} e^{i \beta z}
$$

Here, $\beta$ is real, and $K$ is complex.

The assumed wave form in the periodic media for the second method is given by

$$
E(x, z)=\left(c_{1} e^{i k \alpha x}+c_{2} e^{-i k \alpha x}\right) e^{i k \beta z}
$$

In this case, $\beta$ is complex.

Comparing Equations 42 and 43 , there are some differences.

In Equation 42, the terms in the square brackets form a periodic function. Therefore, the assumed wave form is a periodic function multiplied by a complex exponential in $x$. The propagation constant (in the $z$ direction) is real; hence there is no loss with propagation in the $z$ direction.

In Equation 43, there is no complex exponential in $x$ multiplying the periodic terms in parentheses. Also, the propagation constant (in the $z$ direction) in this case is complex, so there is loss with propagation in the $z$ direction.

In the Bloch analysis, the solution is a confined, lossless mode (assuming a periodic medium that is semi-infinite in extent). In the thin film analysis, the mode is confined, but it is not lossless.

This now brings up the question of which method to use. According to the Floquet theorem, the Bloch wave is the appropriate form for a wave propagating in a periodic medium. Therefore, the appropriateness of the two methods depends on whether or not the layered medium can be considered a periodic semi-infinite medium.

We refer to Reference [CYY77], in which the confined propagation was observed in a Bragg reflection waveguide. Although the oscillatory behavior in the layered Bragg medium could not be resolved in their experimental setup, attenuation ${ }^{1}$ measurements eliminate the possibility that the observed mode is a leaky mode.

Also, calculations show that the $K$ values for such a Bragg reflection waveguide are such that the field has severely decayed after a few periods. Consequently, a

\footnotetext{
${ }^{1}$ There is attenuation, because the Bragg reflector has a finite number of periods.
} 
finite number of periods can be a good approximation to the semi-infinite layered medium, initially assumed for the Bloch waves.

\section{Bloch Wave Implementation}

The electromagnetic analysis of the propagation in a periodic media using Bloch waves has been implemented in Mathematica [Wol91]. The code is shown in Appendix A. The structure modeled in this example is shown in Figure 3 , with parameter values given in Table 1 . Values for $\lambda, n_{1}, n_{2}, n_{g}, n_{a}, t, b$ and $a$ fully describe the structure.

\begin{tabular}{|l|l|}
\hline \hline Parameter & Value \\
\hline$\lambda$ & $0.98010^{-6}$ \\
$n_{1}$ & 2.984 \\
$n_{2}$ & 3.5235 \\
$n_{c}=n_{a}$ & 1 \\
$t$ & to be determined \\
$b$ & $0.069510^{-6}$ \\
$a$ & $0.082110^{-6}$ \\
\hline \hline
\end{tabular}

Table 1: Parameter Values for Example 1

The program has built in functions for the evanescent condition (Equation 13), the left hand side and right hand side of Equation 17, the Bloch wave number (Equation 12) and the effective attenuation constant (Equation 21).

Figure 5 is a plot of $\left|\frac{A+D}{2}\right|-1$ vs. $k \beta$. When the curve in Figure 5 is greater than zero, the Bloch propagation is in the forbidden region. This is required for a guided mode.

Figure 6 , which is a plot of $A+D$ vs. $k \beta$, is useful to check that the correct sign for the square root is used in Equation 6. The sign of the root should be the same as the sign of $A+D$.

For any value of $\beta$ that satisfies the requirement of propagation in the forbidden region in Figure 5, the right hand side of Equation 17 is real. This is seen in Figure 7, which is a plot of the right hand side of Equation $17 \mathrm{vs.} k \beta$. There exists a corresponding confined, lossless mode when Equation 17 is satisfied. Figure 8 shows a combined plot of the RHS and LHS of Equation 17 as a function of guide thickness for $k \beta=3.44$. The intersections correspond to phase thicknesses of $2 \pi$ for the mode propagating with angle $\theta=\arcsin \left[\frac{\beta}{n_{g} / k}\right]$. For these thicknesses, a confined, lossless mode with corresponding $\beta$ value exists. A similar plot for $k \beta=3.2$ is shown in Figure 9 . 


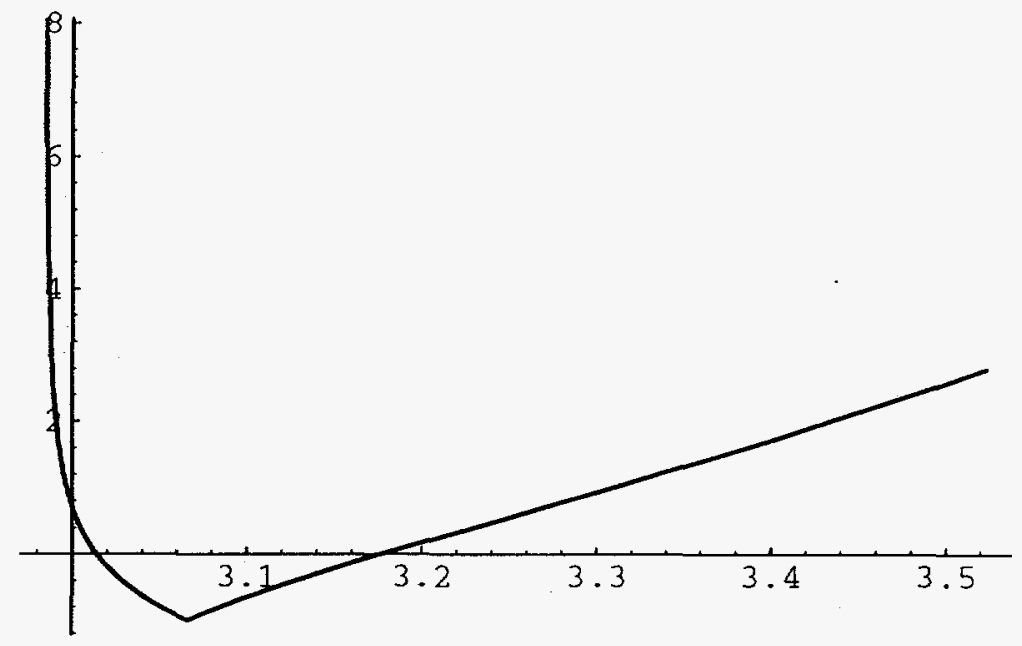

Figure 5: Plot of $\left|\frac{A+D}{2}\right|-1$ vs. $k \beta$

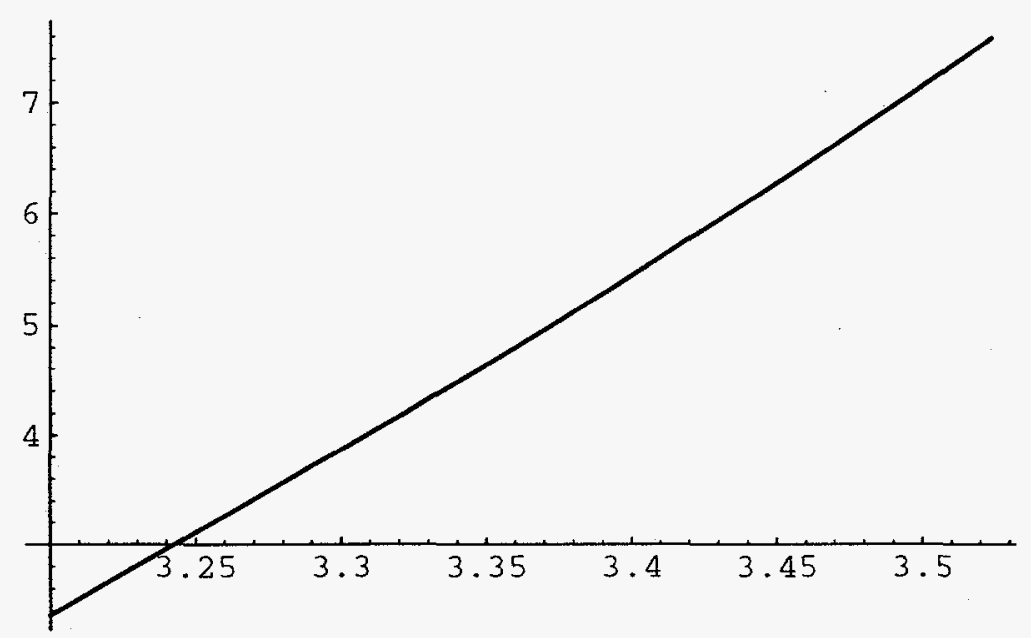

Figure $6: A+D$ vs. $k \beta$ 


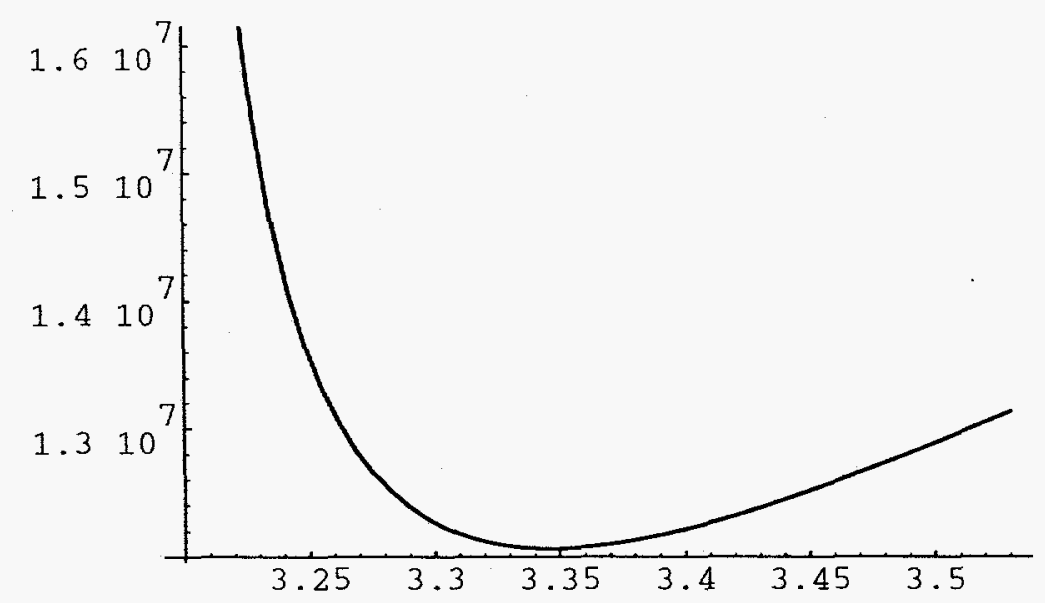

Figure 7: Plot of the right hand side of the dispersion equation vs. $k \beta$. 


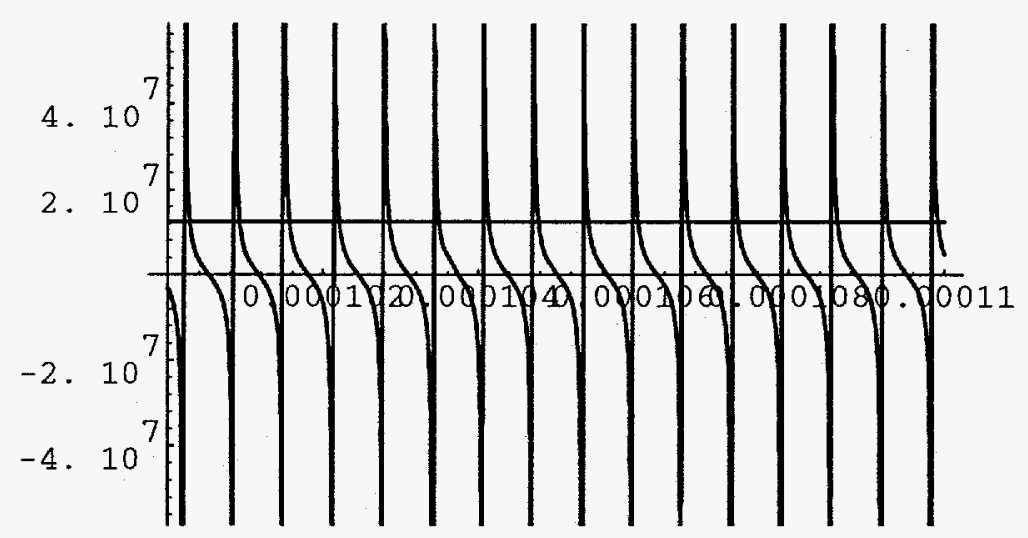

Figure 8: Combined plot of the RHS and LHS of the dispersion equation as a function of guide thickness for $k \beta=3.44$ 


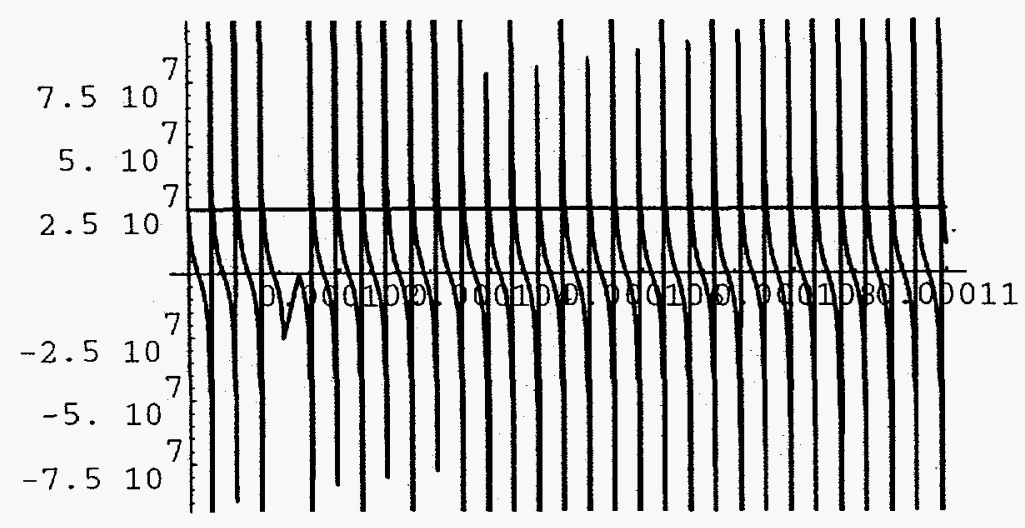

Figure 9: Combined plot of the RHS and LHS of the dispersion equation as a function of guide thickness for $k \beta=3.2$

\section{$5 \quad$ Field Transfer Matrix Approach}

For completeness, code written to implement the unimodular matrix method is included in Appendix B. There are two versions, depending on the structure to be analyzed. In both cases, the thickness and index of each layer must be specified. In one version, each layer is explicitly described. In the other version, which is appropriate for the periodic layered structure in Figure 3, the thicknesses and indices to describe one unit cell are explicitly given, along with the number of unit cells.

The way these implementations work is to provide a function $\chi[\beta]$, which is a complex function of a complex argument. Roots of $\chi[\beta]$ correspond to bound and leaky modes, as described in Sections 2.2.1 and 2.2.3. Roots to this function can be found by plotting the function and graphically looking for the roots, or by using built-in functions in Mathematica.

The correctness of the implementation with explicitly described layers has been verified by applying it to a conventional, 3-layer, dielectric slab waveguide. Results agree with other methods. 


\section{Examples of Confined Lossless Modes in a VCSEL Structures}

In this section we present the results of the Bloch analysis method applied to two examples. A schematic for the structure for both cases is given in Figure 3.

\subsection{Example 1}

The parameters for the first example are given in Table 1.

Five confined, lossless modes of the structure are shown in Table 2. The table shows for each mode the Bloch number, ray angle, and attenuation with varying number, $N$, of unit cells in the periodic medium. To find the modes, the $\beta$ values were first chosen, making sure that Equation 5 is satisfied. Then values for thicknesses that satisfied Equation 17 were found. With this information set, the other values can be calculated.

\begin{tabular}{|l||l|l|l|l|l|}
\hline \hline$k \beta$ & 3.2 & 3.3 & 3.4 & 3.44 & 3.5 \\
\hline thickness $[m]$ & 0.0000993317 & 0.000100015 & 0.000115019 & 0.000100275 & 0.000101343 \\
\hline \hline$K_{i}$ & $3.8836410^{6}$ & $8.4339510^{6}$ & $1.0950210^{7}$ & $1.176410^{7}$ & $1.2862710^{7}$ \\
\hline angle & 65.2561 & 69.4831 & 74.7854 & 77.5016 & 83.379 \\
\hline$\alpha, N=1$ & $1.2819510^{11}$ & $1.2346110^{11}$ & $1.0419810^{11}$ & $1.1812910^{11}$ & $1.1488110^{11}$ \\
\hline$d B / c m, N=1$ & $1.1127310^{10}$ & $1.0716410^{10}$ & $9.0444210^{9}$ & $1.0253610^{10}$ & $9.9716610^{9}$ \\
\hline$\alpha, N=2$ & $2.2960210^{9}$ & $2.2112410^{9}$ & $1.8662310^{9}$ & $2.1157410^{9}$ & $2.0575610^{9}$ \\
\hline$d B / c m, N=2$ & $1.9929510^{8}$ & $1.9193510^{8}$ & $1.6198910^{8}$ & $1.8364710^{8}$ & $1.7859610^{8}$ \\
\hline$\alpha, N=3$ & $4.4289410^{6}$ & $4.2653810^{6}$ & $3.5998910^{6}$ & $4.0811810^{6}$ & $3.9689510^{6}$ \\
\hline$d B / c m, N=3$ & 384432. & 370235. & 312470. & 354247. & 344505. \\
\hline$\alpha, N=4$ & 8271.3 & 7965.86 & 6723. & 7621.85 & 7412.24 \\
\hline$d B / c m, N=4$ & 717.949 & 691.436 & 583.556 & 661.577 & 643.383 \\
\hline$\alpha, N=5$ & 15.4462 & 14.8758 & 12.5548 & 14.2334 & 13.8419 \\
\hline$d B / c m, N=5$ & 1.34073 & 1.29122 & 1.08976 & 1.23546 & 1.20148 \\
\hline$\alpha, N=10$ & 0. & $3.7995210^{-6}$ & 0. & 0. & 0. \\
\hline$d B / c m, N=10$ & 0. & $3.2979910^{-7}$ & 0. & 0. & 0. \\
\hline \hline
\end{tabular}

Table 2: Propagation Information for Guided Modes from Example 1

\subsection{Example 2}

The parameters for the second example are given in Table 3. 


\begin{tabular}{|l|l|}
\hline \hline Parameter & Value \\
\hline$\lambda$ & $0.98010^{-6}$ \\
$n_{1}$ & 2.984 \\
$n_{2}$ & 3.4243 \\
$n_{c}=n_{a}$ & 1 \\
$t$ & to be determined \\
$b$ & $0.071510^{-6}$ \\
$a$ & $0.082110^{-6}$ \\
\hline
\end{tabular}

Table 3: Parameter Values for Example 2

\begin{tabular}{|l||l|l|l|l|l|}
\hline \hline$k \beta$ & 3.2 & 3.3 & 3.3 & 3.4 & 3.5 \\
\hline thickness $[m]$ & 0.000100027 & 0.000100814 & 0.0000924816 & 0.000100185 & 0.000102551 \\
\hline \hline$K_{i}$ & $6.3008910^{6}$ & $9.6006810^{6}$ & $9.6006810^{6}$ & $1.1785910^{7}$ & $1.3530910^{7}$ \\
\hline theta & 65.2561 & 69.4831 & 69.4831 & 74.7854 & 83.379 \\
\hline$\alpha, N=1$ & $1.4040910^{11}$ & $1.3509110^{11}$ & $1.4726310^{11}$ & $1.3194110^{11}$ & $1.2521410^{11}$ \\
\hline$d B / c m, N=1$ & $1.2187510^{10}$ & $1.1725910^{10}$ & $1.2782410^{10}$ & $1.1452510^{10}$ & $1.0868610^{10}$ \\
\hline$\alpha, N=2$ & $3.2862310^{9}$ & $3.1617710^{9}$ & $3.4466410^{9}$ & $3.0880410^{9}$ & $2.930610^{9}$ \\
\hline$d B / c m, N=2$ & $2.8524410^{8}$ & $2.7444110^{8}$ & $2.9916810^{8}$ & $2.6804210^{8}$ & $2.5437610^{8}$ \\
\hline$\alpha, N=3$ & $6.4310410^{6}$ & $6.1874710^{6}$ & $6.7449510^{6}$ & $6.0431910^{6}$ & $5.7350910^{6}$ \\
\hline$d B / c m, N=3$ & 558214. & 537073. & 585462. & 524549. & 497806. \\
\hline$\alpha, N=4$ & 12010.7 & 11555.8 & 12597. & 11286.3 & 10710.9 \\
\hline$d B / c m, N=4$ & 1042.53 & 1003.04 & 1093.42 & 979.655 & 929.708 \\
\hline$\alpha, N=5$ & 22.4293 & 21.5798 & 23.5241 & 21.0766 & 20.002 \\
\hline$d B / c m, N=5$ & 1.94686 & 1.87313 & 2.04189 & 1.82945 & 1.73618 \\
\hline$\alpha, N=10$ & 0. & 0. & 0. & 0. & 0. \\
\hline$d B / c m, N=10$ & 0. & 0. & 0. & 0. & 0. \\
\hline \hline
\end{tabular}

Table 4: Propagation Information for Guided Modes from Example 2 
Table 4 shows the same type of information as in Table 2 . However, instead of showing five different $k \beta$ values, the second and third columns refer to the same mode, but with a different guide thickness.

\subsection{Discussion}

Tables 2 and 4 show that various confined, "lossless" modes can be supported by a structure such as in Figure 3, depending on the guide thickness.

The imaginary part of the Bloch constant, $K_{i}$, gives an indication of how fast the Bloch wave is attenuated in the direction of the gradient of the periodic medium. The smaller the value of $K_{i}$, the further away from the guide-periodic medium boundary the Bloch wave can be seen. Consequently, for smaller $K_{i}$, a larger number of unit cells in the periodic medium are required to make the Bloch analysis valid.

It is seen that as the number of unit cells, $N$, in the periodic medium increases, the effective attenuation decreases. This is to be expected, because as $N$ increases, the reflectivity increases, and more energy is reflected back into the guide at the guide-periodic medium boundary.

The effective attenuation is also a function of the thickness of the guide. For an imperfect reflector, $(R<1)$, each "bounce" in the guide corresponds to energy going into the periodic medium. For a given mode and a given length along the guide, there are fewer bounces in a thicker guide. Therefore, the effective attenuation is less for a thicker guide, assuming no scattering in the guide.

Although it is difficult to make exact comparisons because of different guide thicknesses, the effective attenuations for Example 2 are greater than for Example 1. This makes sense, because the distributed Bragg reflector in Example 2 has a smaller $\Delta n$ between the two layers of a unit cell and, hence, is not as good a reflector as the DBR in Example 1.

\section{$7 \quad$ Waveguide Interconnected VCSELs}

As we have seen in the previous section, various confined, lossless modes can be supported by a structure as in Figure 3, depending on the guide thickness. For a fixed guide thickness, a discrete number of confined, lossless modes are supported. Figure 10 is a plot of the LHS and RHS of Equation 17 as a function of $k \beta$ for the structure described in Example 1 and a guide thickness of $100 \mu \mathrm{m}$. Intersections of the two curves correspond to supported modes. Each of these modes propagates with a different propagation constant, and corresponding angle in the guide.

As in Figure 1, a diffractive optical element (DOE) could be placed on the guidecover interface, to couple the laser light into one of the confined, lossless modes 


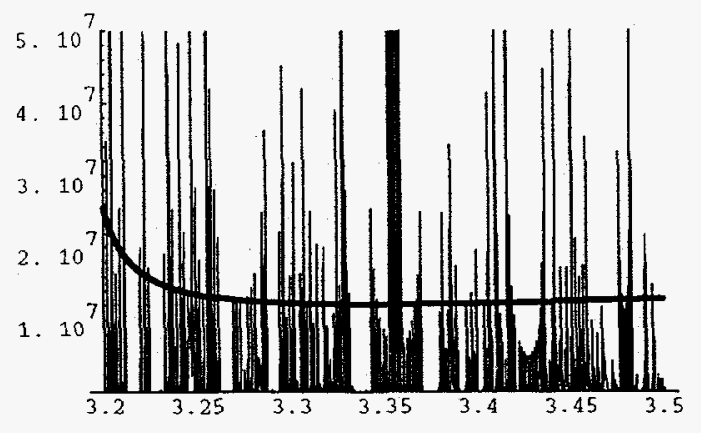

Figure 10: Solutions to the dispersion equation for the structure described in Example 1 and a guide thickness of $100 \mu \mathrm{m}$.

described above. The DOE could also be designed so that it couples into a number of the supporting modes.

Different lasers with different wavelengths can also be grown on the same substrate. For each color, there are likely supported confined, lossless modes. This is demonstrated in Figure 11, which shows the dispersion equation for the structure in Example 1, with $\lambda=.98 \times 10^{-6}, \lambda=.99 \times 10^{-6}$ and $\lambda=1.3 \times 10^{-6}$.

DOEs are sensitive to both incident angle and wavelength. By placing other DOEs along the cover-guide boundary, the output laser light may be directed in a large variety of ways. This could be used to implement broadcast or selective coupling among VCSELs on the same substrate.

In addition, this type of guiding can allow the light from a number of VCSELs to be easily coupled out into a single fiber, whose dimensions are the same as the substrate (guide) thickness. This light could be composed of different modes, corresponding to different modes from the same VCSEL and/or from different VCSELs.

There are many possible scenarios in which waveguide interconnected VCSELs could be useful. Using the tools presented in the previous sections, system concepts incorporating waveguide interconnected VCSELs can be evaluated. The tools are also useful in making the design calculations for specific implementations. 

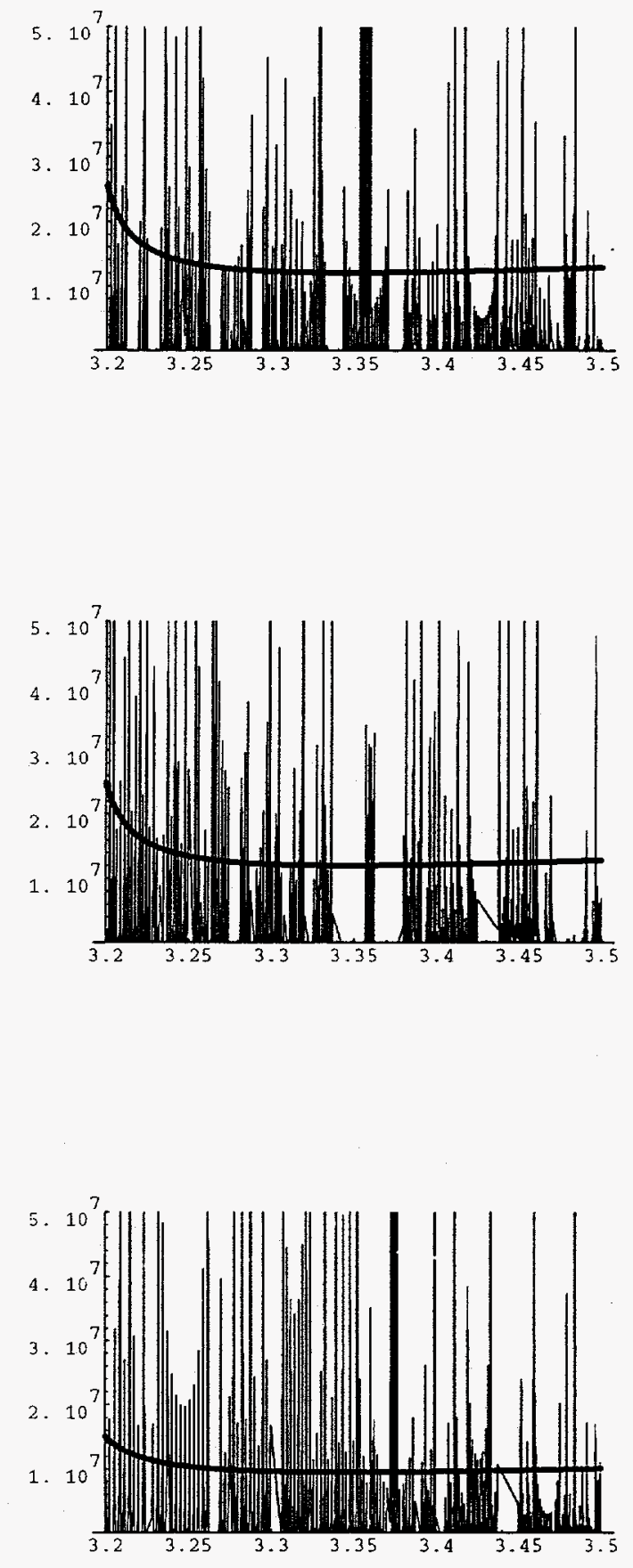

Figure 11: Solutions to the dispersion equation for a) $\lambda=.98 \times 10^{-6}$; b) $\lambda=.99 \times 10^{-6}$; and c) $\lambda=1.3 \times 10^{-6}$ for guide thickness of $100 \mu \mathrm{m}$. 


\section{Conclusion}

In this report the feasibility of using the DBR grown on the substrate for a VCSEL to provide waveguiding within the substrate has been shown. The guided modes in such a structure are confined, but not strictly lossless, due to the finite extent of a manufactured DBR.

Before determining the feasibility of waveguide interconnected VCSELs, two analysis methods were presented and evaluated for their applicability to this problem. Both methods were implemented in Mathematica. The Bloch wave method was shown to be the appropriate analysis method for the structure under consideration.

There are many possible uses of waveguide interconnected VCSELs. The tools and analysis presented in this report can be used to evaluate such system concepts and to do detailed design calculations.

\section{A Bloch Wave Method}

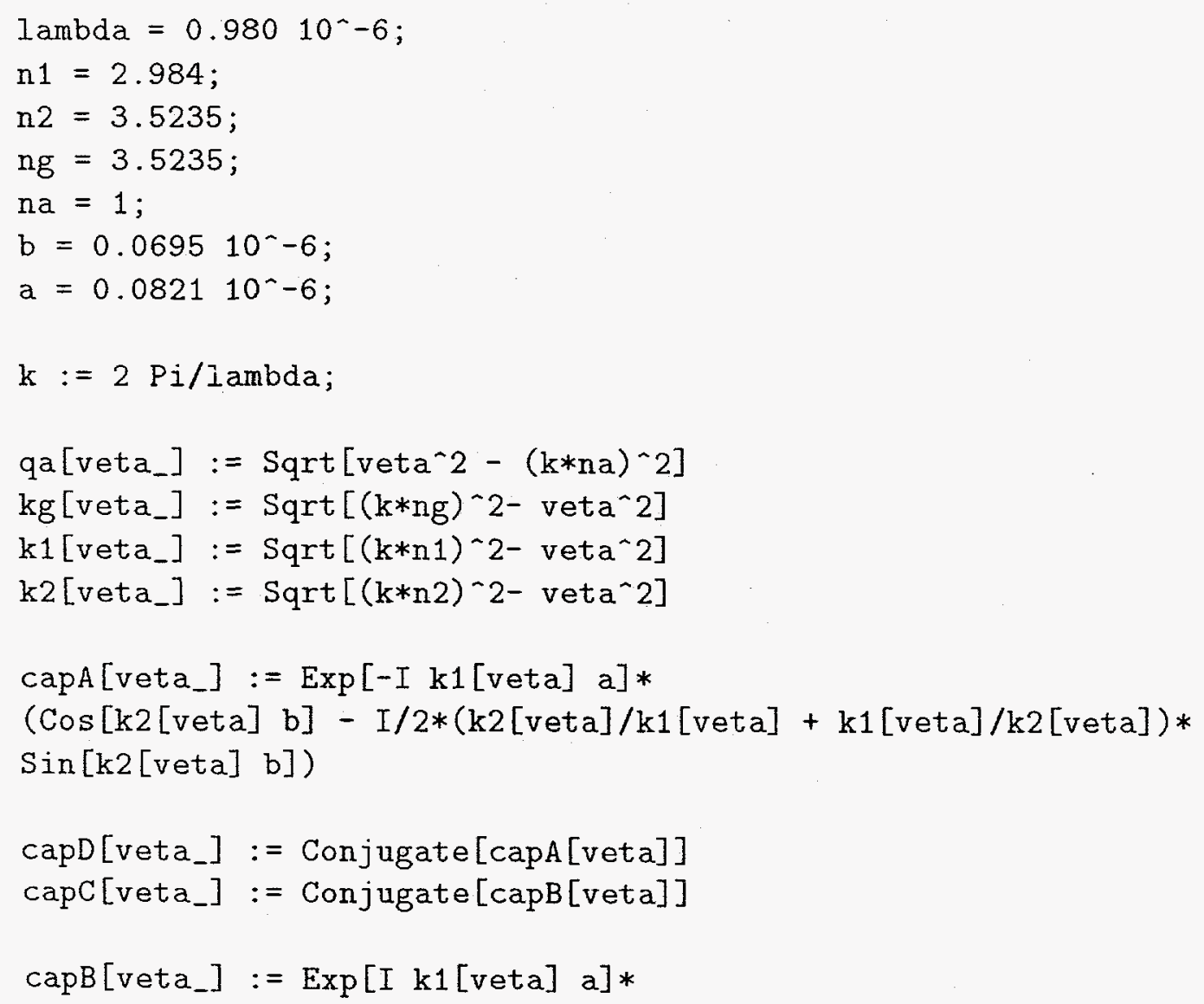




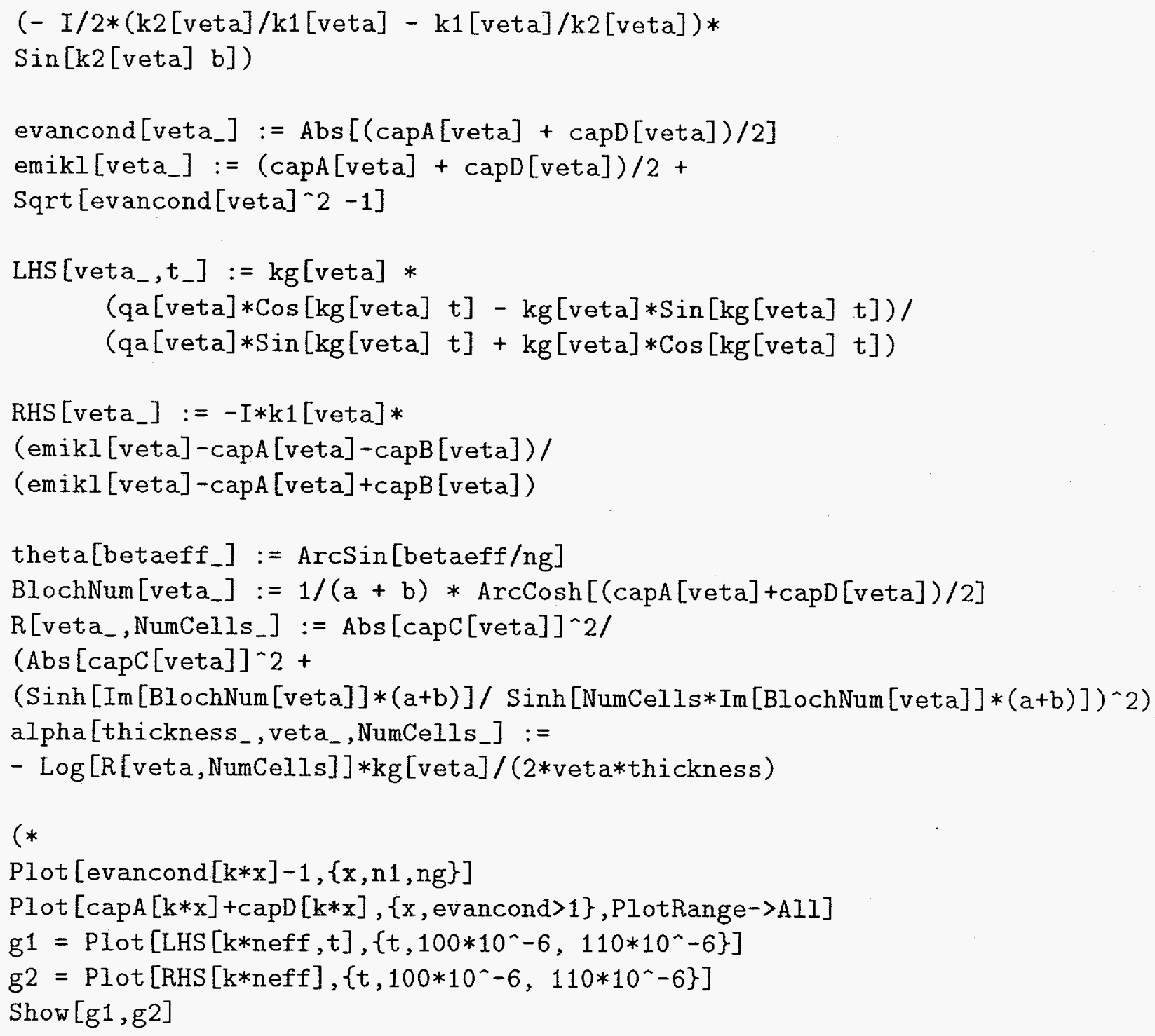




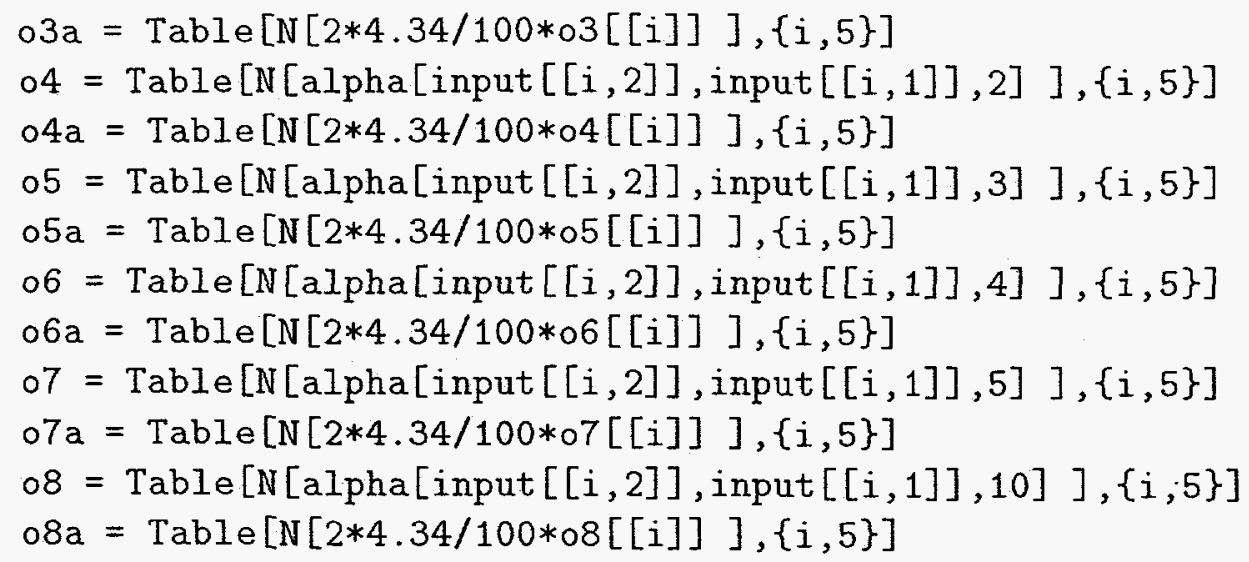

NumberForm $[\{$ oobeta, o0thick, $\operatorname{Re}[01], 02,03,03 \mathrm{a}, 04$, $04 \mathrm{a}, 05,05 \mathrm{a}, 06,06 \mathrm{a}, 07,07 \mathrm{a}, 08, \mathrm{~N}[08 \mathrm{a}]\},\{6,4\}]$

PutAppend [OutputForm [TableForm [\%]] , "case2table"]

$\mathrm{g} 1=\mathrm{Plot}[\operatorname{RHS}[\mathrm{k} * \mathrm{x}],\{\mathrm{x}, 3.2,3.5\}, \mathrm{PlotStyle}->$ Thickness $[0.01]]$

$t=10010^{-}-6$

$\mathrm{g} 2=\mathrm{Plot}[\operatorname{LHS}[\mathrm{k} * \mathrm{x}, \mathrm{t}],\{\mathrm{x}, 3.2,3.5\}, \mathrm{PlotStyle}->$ Thickness $[0.001]]$

\section{B Field Transfer Matrix Approach}

\section{B.1 Explicit Layer Definition}

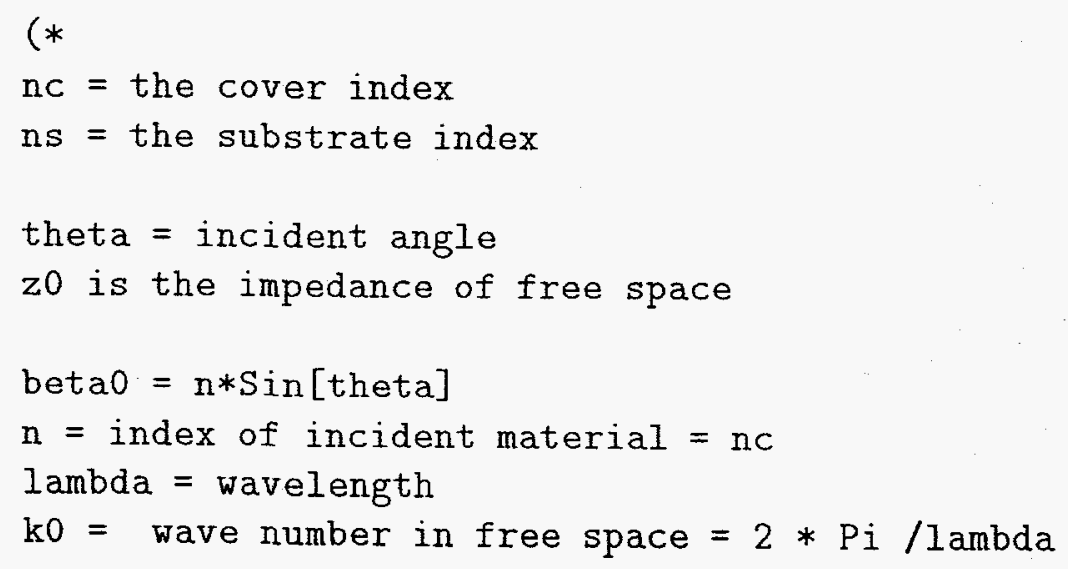




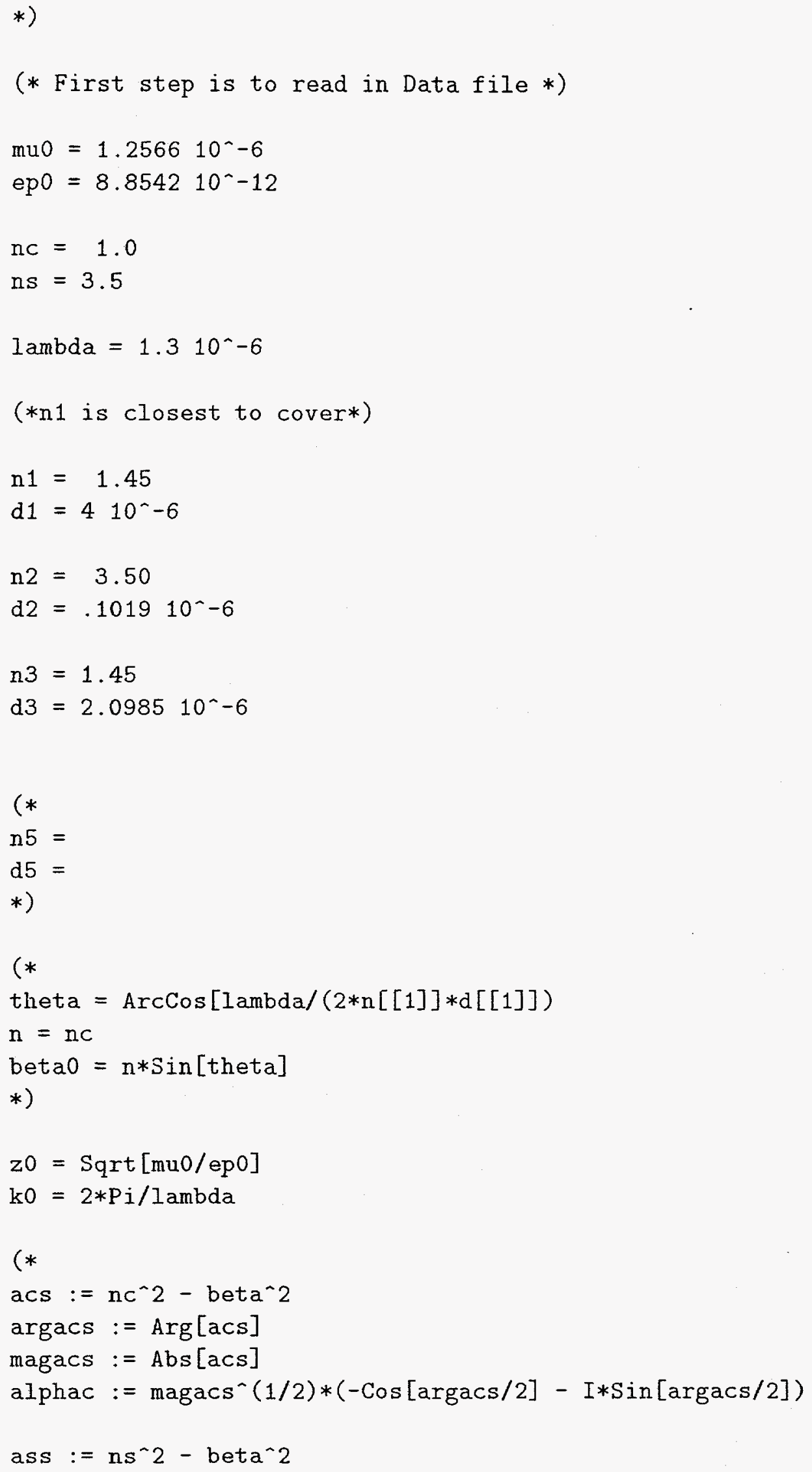




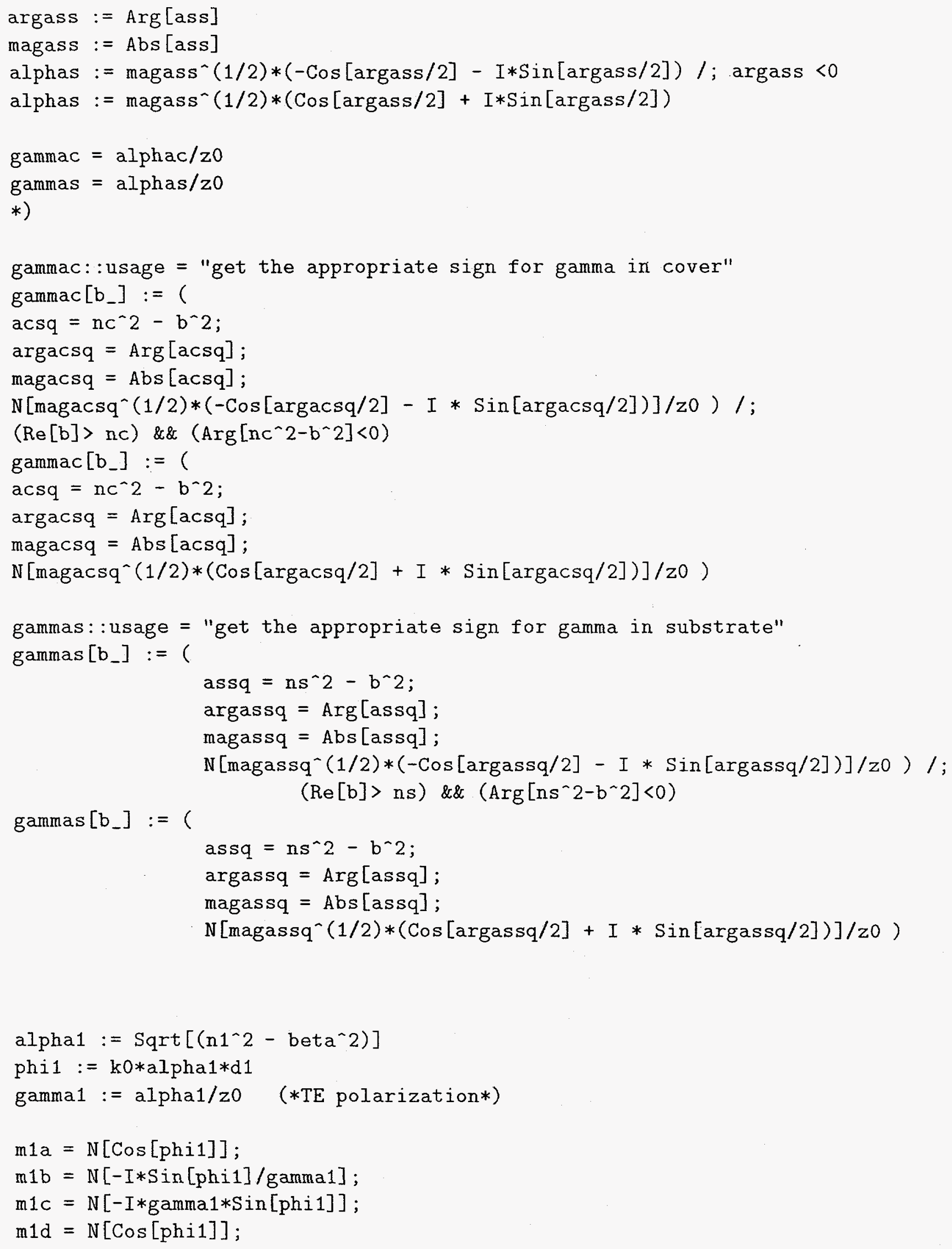




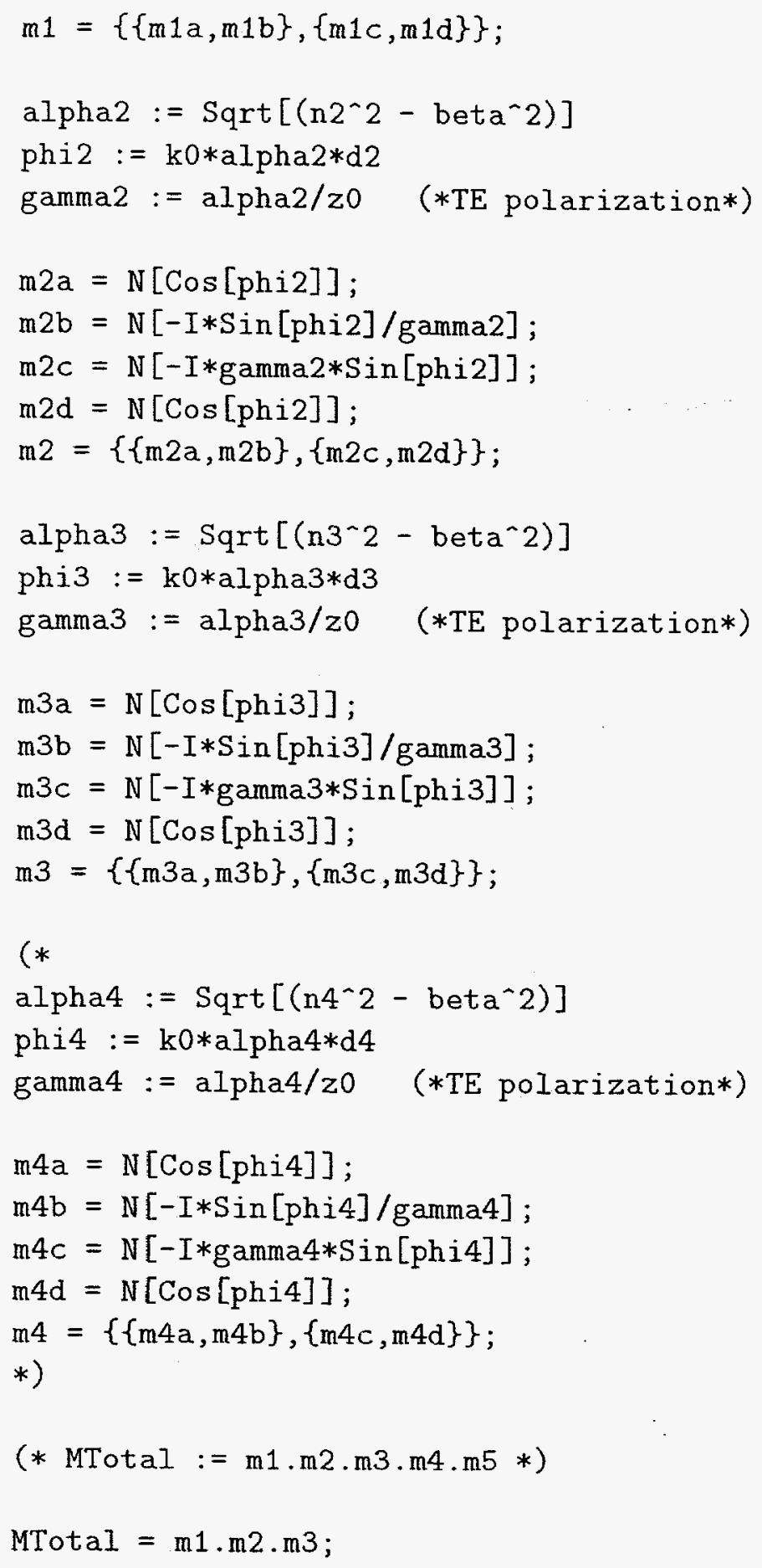


(*

Plot3D $[$ Abs $[P \operatorname{Plotxi}[\mathrm{x}, \mathrm{y}]],\{\mathrm{x}, 1,1.5\},\{\mathrm{y}, 0,0.1\}, \mathrm{PlotRange}->\{0,10\}]$

Plot3D $[\operatorname{Re}[P \operatorname{lot} x i[x, y]],\{x, 1,1.5\},\{y, 0,0.1\}]$

$\mathrm{P} \operatorname{lot} 3 \mathrm{D}[\operatorname{Im}[\mathrm{P} \operatorname{lot} \mathrm{xi}[\mathrm{x}, \mathrm{y}]],\{\mathrm{x}, 1,1.5\},\{\mathrm{y}, 0,0.1\}]$

*)

FindRoot $[\{\operatorname{Re}[P \operatorname{lot} x i[x, y]]==0, \operatorname{Im}[\operatorname{Plotxi}[x, y]]==0\},\{x, 1,1.5\},\{y, 0,0.5\}$, MaxIterations $->100]$

Plot $[R e[P I o t x i[x, 0]],\{x, 1.3,1.44\}]$

$P \operatorname{Plot}[\operatorname{Im}[P \operatorname{lot} x i[x, 0]],\{x, 1.3,1.44\}]$

Plot $[A b s[P l o t x i[x, 0]],\{x, 1.3,1.44\}]$

\section{B.2 Periodic Layer Definition}

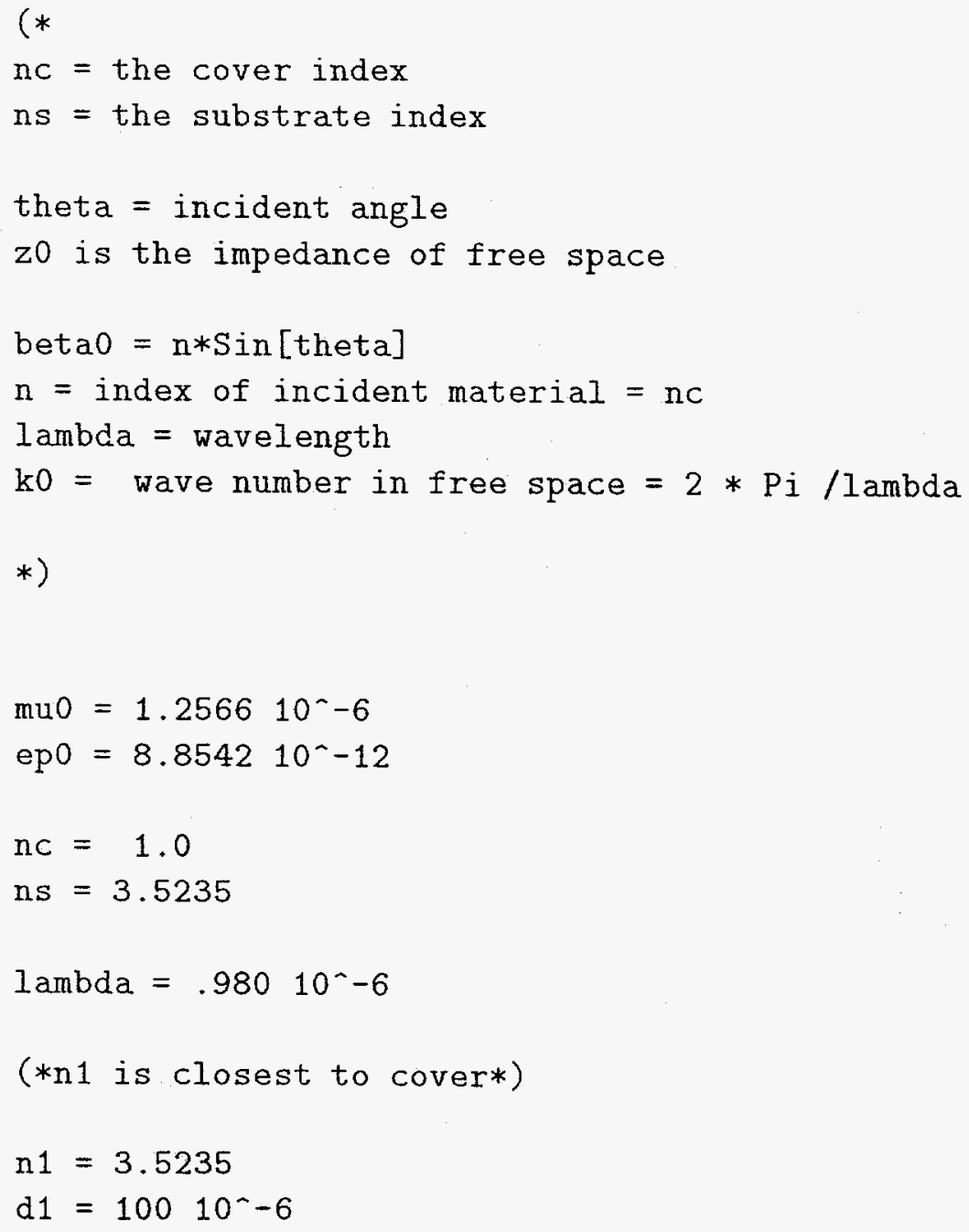




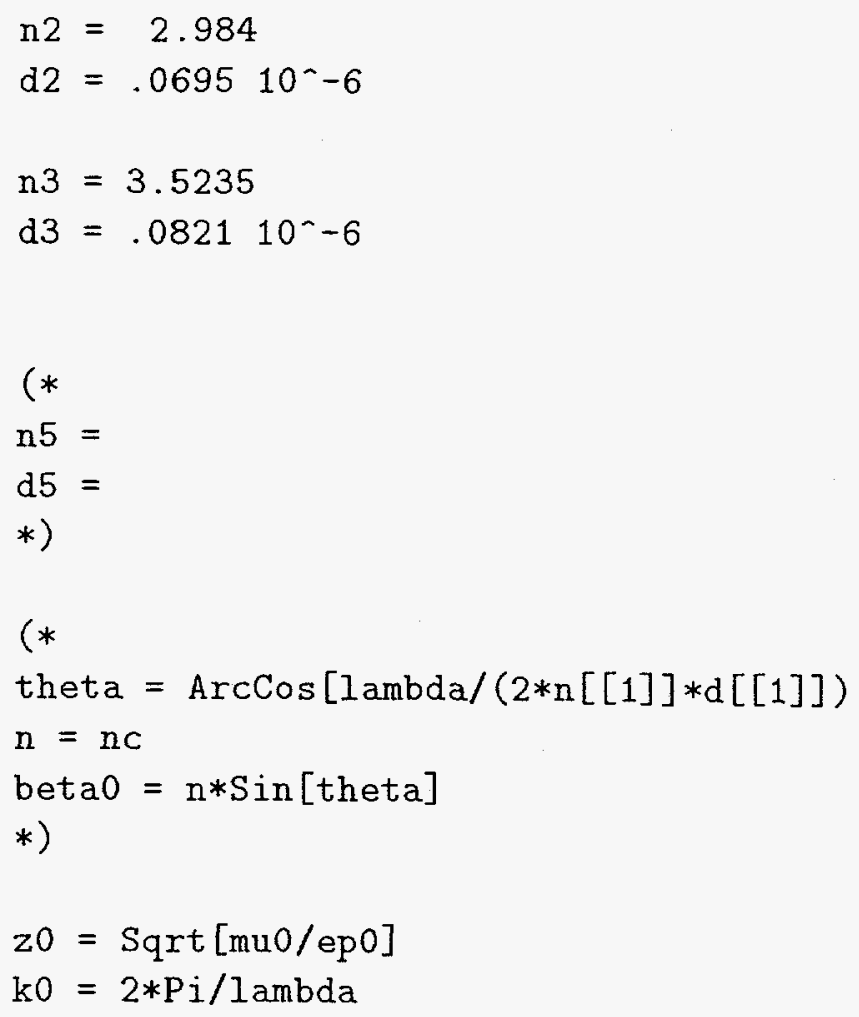




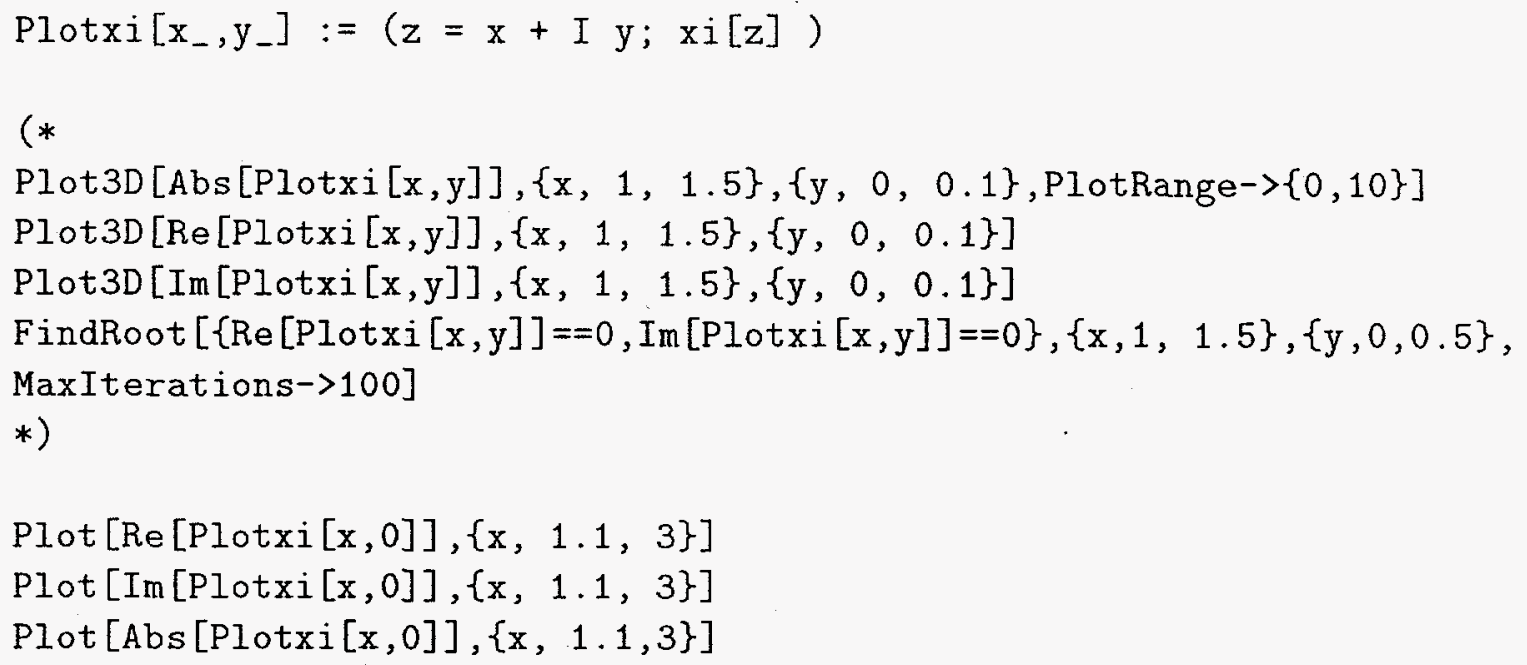

\section{References}

[BKSI88] T. Baba, Y. Kokubun, T. Sakaki, and K. Iga. Loss reduction of an arrow waveguide in shorter wavelength and its stack configuration. Journal of Lightwave Technology, 6(9):1440-1445, September 1988.

[Blo28] F. Bloch. Physics, 52:555, 1928.

[BW75] M. Born and E. Wolf. Principles of Optics. Pergamon, 1975.

[CH84] John Chilwell and Ian Hodgkinson. Thin-films field-transfer matrix theory of planar multilayer waveguides and reflection from prism-loaded waveguides. JOSA A, 1(7):742-753, July 1984.

[CYY77] A. Cho, A. Yariv, and P. Yeh. Observation of confined propagation in bragg waveguides. Applied Physics Letters, 30(9):471-472, May 1977.

[DKK86] M. Duguay, Y. Kokubun, and T. Koch. Antiresonanat reflecting optical waveguides in sio2-si multilayer structures. Applied Physics Letters, 49(1):13-15, July 1986.

[JCF89] W. Jiang, J. Chrostowski, and M. Fontaine. Analysis of arrow waveguides. Optics Communications, 72(3,4):180-186, July 1989.

$\left[\mathrm{KKB}^{+} 87\right]$ T. Koch, U. Koren, G. Boyd, P. Corvina, and M. Duguay. Antiresonanat reflecting optical waveguides for iii-v integrated optics. Electronics Letters, 23(5):244-245, February 1987.

[KUC90] J. Kubica, D. Uttamchandani, and B. Culshaw. Modal propagation within arrow waveguides. Optics Communications, 78(2):133-136, August 1990.

[Mar91] D. Marcuse. Theory of Dielectric Optical Waveguides. Academic Press, second edition, 1991. 
[Tie71] P. K. Tien. Light waves in thin films and integrated optics. Applied Optics, 10(11):2395-2413, November 1971.

[Wol91] S. Wolfram. Mathematica: A System for Doing Mathematics by Computer. Addison-Wesley, 2nd edition, 1991.

[Yeh88] Pochi Yeh. Optical Waves in Layered Media. J. Wiley and Sons, 1988.

[YY76] Pochi Yeh and Amnon Yariv. Bragg reflection waveguides. Optics Communications, 19(3):427-430, December 1976.

[YY77] Amnon Yariv and Pochi Yeh. Electromagnetic propagation in periodic stratified media. ii. birefringence, phase matching and $\mathrm{x}$-ray lasers. Journal of the Optical Society of America, 67(4):438-448, April 1977.

[YY84] Amnon Yariv and Pochi Yeh. Optical Waves in Crystals. J. Wiley and Sons, 1984.

[YYH77] Pochi Yeh, Amnon Yariv, and CS Hong. Electromagnetic propagation in periodic stratified media. i. general theory. Journal of the Optical Society of America, 67(4):423-438, April 1977. 


\section{DISTRIBUTION:}

1 MS 9018 Central Technical Files, 8523-2

$5 \quad$ MS 0899 Technical Library, 4414

2 MS 0619 Review and Approval Desk, 12630

For DOE/OSTI

1 MS $0843 \quad$ I. A. Erteza, 2425 